\title{
A radio census of nuclear activity in nearby galaxies
}

\author{
M. E. Filho ${ }^{1,2,3}$, P. D. Barthel ${ }^{3}$, and L. C. $\mathrm{Ho}^{4}$ \\ 1 Centro de Astrofísica da Universidade do Porto, Rua das Estrelas, 4150 - 762 Porto, Portugal \\ e-mail:mfilho@astro.up.pt \\ 2 Istituto di Radioastronomia, CNR, via P. Gobetti, 101, 40129 Bologna, Italy \\ 3 Kapteyn Astronomical Institute, PO Box 800, 9700 AV Groningen, The Netherlands \\ 4 The Observatories of the Carnegie Institution of Washington, 813 Santa Barbara Street, Pasadena, CA 91101, USA
}

Received 10 November 2005 / Accepted 30 December 2005

\section{ABSTRACT}

In order to determine the incidence of black hole accretion-driven nuclear activity in nearby galaxies, as manifested by their radio emission, we have carried out a high-resolution Multi-Element Radio-Linked Interferometer Network (MERLIN) survey of LINERs and composite LINER/HII galaxies from a complete magnitude-limited sample of bright nearby galaxies (Palomar sample) with unknown arcsecond-scale radio properties. There are fifteen radio detections, of which three are new subarcsecond-scale radio core detections, all being candidate AGN. The detected galaxies supplement the already known low-luminosity AGN - low-luminosity Seyferts, LINERs and composite LINER/HII galaxies - in the Palomar sample. Combining all radio-detected Seyferts, LINERs and composite LINER/HII galaxies (LTS sources), we obtain an overall radio detection rate of $54 \%$ (22\% of all bright nearby galaxies) and we estimate that at least $\sim 50 \%(\sim 20 \%$ of all bright nearby galaxies) are true AGN. The radio powers of the LTS galaxies allow the construction of a local radio luminosity function. By comparing the luminosity function with those of selected moderate-redshift AGN, selected from the 2dF/NVSS survey, we find that LTS sources naturally extend the RLF of powerful AGN down to powers of about 10 times that of Sgr A*.

Key words. galaxies: active - galaxies: nuclei - galaxies: luminosity function, mass function

\section{Introduction}

The search for low-luminosity active galactic nuclei (LLAGN) in nearby galaxies has been the subject of many optical surveys. Results show that nuclear activity may be a common phenomenon. The Palomar survey (Ho et al. 1995, 1997a,b) has been very useful in this regard by providing a sensitive magnitude-limited $\left(B_{\mathrm{T}}<12.5 \mathrm{mag}\right)$ sample of almost 500 bright nearby galaxies. About half of the sources are emission-line nuclei, classified as Seyferts, LINERs or composite LINER/HII galaxies, the last category displaying both LINER and HII properties. However, characterizing the powering mechanisms of the sources is not straightforward, particularly in low-luminosity sources. Many of these galaxies possess circum-nuclear star-forming regions which blend with and may even drown out the presence of a weak active galactic nucleus (AGN).

Optimally it is necessary to pick spectral regions where the contrast between any hypothetical LLAGN component and circum-nuclear stellar component is maximized. X-rays are very useful in this regard as shown by the hard X-ray studies of LLAGN (Terashima et al. 2000b; Terashima et al. 2000a; Ho et al. 2001; Terashima \& Wilson 2003). In the absence of spectral AGN signatures such as a Seyfert or quasar-type continuum or broad emission lines, radio observations can offer an alternative method for determining the LLAGN incidence in nearby galaxies. Measurements of radio flux, compactness, radio spectral index ${ }^{1}$ and brightness temperatures provide the necessary diagnostic tools for determining the nature of the radio emission.

Several important radio surveys have been conducted on the magnitude-limited Palomar bright nearby galaxy sample (Ho et al. 1995, 1997a,b), revealing a large fraction of radio cores, not only in ellipticals but also in bulge-dominated spirals. In a recent Very Large Array (VLA) 5 and $1.4 \mathrm{GHz}$, 1 " resolution survey of the low-luminosity Seyferts of the Palomar sample (Ho \& Ulvestad 2001; HU01 hereafter), it was found that over $80 \%$ of the sources harbour a radio core. In a distance-limited sample of low-luminosity Seyferts, LINERs and composite LINER/HII galaxies observed with the VLA at $15 \mathrm{GHz}, 0.25$ resolution (Nagar et al. 2000, 2002; VLA/N00 and VLA/N02 hereafter; Nagar et al. 2005; VLA/N05 hereafter), it was found that $\sim 40 \%$ of the objects harbour subarcsecond-scale compact radio cores. A similar study with the VLA, at $8.4 \mathrm{GHz}, 2$ ". 5 resolution of all the composite LINER/HII galaxies in the Palomar sample has been presented in Filho et al. (2000, 2002a), revealing radio cores in $\sim 25 \%$ of the sample sources. However, although

\footnotetext{
${ }^{1} F_{v} \propto v^{-\alpha}$ throughout.
} 
the radio core emission in these sources is consistent with the presence of a LLAGN, we cannot exclude a stellar origin from the brightness temperature figures $\left(T_{\mathrm{B}} \lesssim 10^{5} \mathrm{~K}\right.$; Condon 1992) obtained at these resolutions. As conclusive judgement requires Very Long Baseline (VLBI)-resolution, multi-wavelength Very Long Baseline Array (VLBA) and European Very Long Baseline Interferometer Network (EVN) observations have been obtained for selected subsamples of low-luminosity Seyferts, LINERs and composite LINER/HII galaxies that showed arcsecond- or subarcsecond-scale radio cores (Falcke et al. 2000; F00 hereafter; Nagar et al. 2002; VLBA/N02 hereafter; Nagar et al. 2005; VLBA/N05 hereafter; Ulvestad \& Ho 2001b; Filho et al. 2002b; Filho et al. 2004; Anderson et al. 2004; AU04 hereafter). In sources with subarcsecond- or arcsecond-scale radio peak emission above $2.5 \mathrm{mJy}$, results reveal a $100 \%$ detection rate of high-brightness temperature $\left(T_{\mathrm{B}} \gtrsim 10^{8} \mathrm{~K}\right)$, compact, flat spectrum $(\alpha<0.5)$ radio cores, enforcing the LLAGN scenario for the radio emission (VLBA/N05; see also Ulvestad \& Ho 2001b; Filho et al. 2002b; Filho et al. 2004; AU04). Their low radio luminosities suggest we are probing the very faint end of the AGN population.

Unambiguously determining the physical nature of the nearby galaxy radio cores is more than of mere phenomenological interest. If they truly contain an accretion-powered nucleus, then they obviously need to be included in the AGN population. Their non-trivial numbers impact on several astrophysical problems ranging from the cosmological evolution of the AGN luminosity function to their contribution to the X-ray background.

The present paper deals with high-resolution radio-imaging of LLAGN, carried out with the Jodrell Bank Multi-Element Radio-Linked Interferometer Network (MERLIN), completing the radio census of nuclear activity in the Palomar galaxy survey of Ho et al. (1997a).

\section{Source selection}

The target sources were taken from the Palomar survey of bright nearby galaxies (Ho et al. 1997a). The Palomar sample contains a total of 417 emission-line objects (486 galaxies in total), of which 206 are stellar-powered HII nuclei. Among the remaining sources, 52 are classified as low-luminosity Seyfert galaxies, 94 as LINERs and 65 as composite LINER/HII galaxies, many of which may be AGN-powered. After the extensive radio studies described above which targeted all lowluminosity Seyfert and part of the LINERs and composite LINER/HII galaxies in the Palomar sample, we have selected the remaining (68) LINERs or composite sources (plus one Seyfert and one HII nucleus; see Table 1) having either unknown radio properties or known radio emission in excess of $2 \mathrm{mJy}$ on arcsecond-scales. Aiming to detect (weak) radio emission on subarcsecond scales, the sources were observed with the Jodrell Bank MERLIN, at $5 \mathrm{GHz}, 0.1$ resolution. These MERLIN observations were intended as a filter for follow-up, high-resolution EVN observations, which should increase the brightness temperature figures to the necessary $>10^{5} \mathrm{~K}$ (Condon 1992) level and thereby unambiguously determine the nature of the radio emission. In Table 1 we summarize the properties of the 70 sources observed with MERLIN.

\section{Observations and data reduction}

The $5 \mathrm{GHz}$ MERLIN observations were obtained during 2001 October 18-20, with a $15 \mathrm{MHz}$ bandwidth. Defford, Cambridge, Knockin, Darnhall and Tabley telescopes were used, yielding a resolution of about 0.1 at $5 \mathrm{GHz}$. Typically four $8 \mathrm{~min}$ scans of the sources were interspersed with 2 min scans of the respective phase calibrators, for a total integration time of about $25 \mathrm{~min}$ on each target source. 3C 286, the primary flux calibrator, was observed twice during the observing run. Initial calibration, reduction and imaging of the MERLIN data was performed by Garrington and Richards using the AIPS pipeline at Jodrell Bank.

\section{Radio properties}

Of the 70 objects observed with MERLIN, fifteen objects were detected above the $5 \sigma\left(\sim 0.5 \mathrm{mJy}^{\text {beam }}{ }^{-1}\right)$ threshold. Three of these are new radio core detections on subarcsecond scales. With the exception of the composite galaxy NGC 4321, all of the detected sources are spectroscopically classified as LINERs. All detected sources except for NGC 4293 and NGC 4321 show compact, unresolved cores near the phase center, coincident with the optical nucleus.

In Table 2 we summarize the radio parameters for the MERLIN-detected sources. The rms noise level was measured in a source-free region using the AIPS task IMSTAT. MERLIN radio maps of the detected sources (excluding NGC 4321) are shown in Figs. 1-4. The map for NGC 4321 can be seen in Fig. 5, along with the detected background sources. Contour levels are $\mathrm{rms} \times(-3,3,6,12,24,48,96,192)$.

Table 3 includes the model fits to the detected radio components. The AIPS task IMFIT was used to fit bi-dimensional Gaussians to the brightness peaks in each radio component. The brightness temperature has been calculated using the formula (Weedman 1986):

$T_{\mathrm{B}}=4.9 \times 10^{7}\left(\frac{F_{v}}{\mathrm{mJy}}\right)\left(\frac{\theta_{\text {major }} \times \theta_{\text {minor }}}{\mathrm{mas}^{2}}\right)^{-1}\left(\frac{v}{5 \mathrm{GHz}}\right)^{-2}$,

where $F_{v}$ is the peak flux density of the sources and $\theta_{\text {major }}$ and $\theta_{\text {minor }}$ refer to the major and minor axis of the Gaussian beam (Table 2).

Below we give a source description, quoting values from surveys such as the NVSS, FIRST, VLA/N05 and VLBA/N05 (see Table 4 for details). Sources are said to be "compact" if the FIRST flux density is $>50 \%$ the NVSS value, otherwise the source is said to be resolved. Furthermore, we assume that any radio variability is small compared to the total NVSS flux. We also present a brief discussion on detected background sources (Fig. 5).

NGC 2768: this source has a NVSS (14.5 mJy) and a FIRST (12.3 $\mathrm{mJy}$ ) radio detection, denoting that the source is compact on arcsecond scales. NGC 2768 also has an EVN (7 mJy; 
Table 1. Target MERLIN sources. Column 1: source name; Cols. 2 and 3: optical position from NASA/IPAC Extragalactic Database (NED); Col. 4: adopted distance from Ho et al. (1997a) with $H_{0}=75 \mathrm{~km} \mathrm{~s}^{-1} \mathrm{Mpc}^{-1}$; Col. 5: spectral type from Ho et al. (1997a). L = LINERs, S = Seyferts, and T = composite LINER/HII galaxies. Colons refer to uncertain (:) or highly uncertain (::) spectral classification. The number 1.9 refers to the presence of broad $\mathrm{H} \alpha$ emission and 2 refers to the absence of broad $\mathrm{H} \alpha$ emission; Col. 6: hubble type from Ho et al. (1997a).

\begin{tabular}{|c|c|c|c|c|c|}
\hline $\begin{array}{l}\text { Galaxy } \\
\text { (1) }\end{array}$ & $\begin{array}{c}\mathrm{RA}(\mathrm{J} 2000) \\
(\mathrm{h} \mathrm{m} \mathrm{s}) \\
(2)\end{array}$ & $\begin{array}{c}\operatorname{Dec}(\mathrm{J} 2000) \\
\left({ }^{\circ},{ }^{\prime \prime}\right) \\
(3)\end{array}$ & $\begin{array}{c}D \\
(\mathrm{Mpc}) \\
(4)\end{array}$ & $\begin{array}{l}\text { Spectral type } \\
\text { (5) }\end{array}$ & $\begin{array}{l}\text { Hubble type } \\
\text { (6) }\end{array}$ \\
\hline IC 356 & 040746.9 & +694845 & 18.1 & $\mathrm{~T} 2$ & SA(s)ab pec \\
\hline IC 520 & 085342.2 & +732927 & 47.0 & T2: & $\mathrm{SAB}(\mathrm{rs}) \mathrm{ab}$ ? \\
\hline NGC 428 & 011255.6 & +005854 & 14.9 & L2/T2: & $\mathrm{SAB}(\mathrm{s}) \mathrm{m}$ \\
\hline NGC 488 & 012146.8 & +051525 & 29.3 & $\mathrm{~T} 2::$ & $\mathrm{SA}(\mathrm{r}) \mathrm{b}$ \\
\hline NGC 521 & 012433.8 & +014352 & 67.0 & T2/H: & $\mathrm{SB}(\mathrm{r}) \mathrm{bc}$ \\
\hline NGC 718 & 015313.3 & +041144 & 21.4 & L2 & $\mathrm{SAB}(\mathrm{s}) \mathrm{a}$ \\
\hline NGC 777 & 020014.9 & +312546 & 66.5 & S2/L2:: & E1 \\
\hline NGC 841 & 021117.3 & +372950 & 59.5 & L1.9: & $\left(\mathrm{R}^{\prime}\right) \mathrm{SAB}(\mathrm{s}) \mathrm{ab}$ \\
\hline NGC 1169 & 030334.7 & +462309 & 33.7 & L2 & $\mathrm{SAB}(\mathrm{r}) \mathrm{b}$ \\
\hline NGC 1961 & 054204.8 & +692243 & 53.1 & L2 & $\mathrm{SAB}(\mathrm{rs}) \mathrm{c}$ \\
\hline NGC 2336 & 072703.7 & +801042 & 2.9 & $\mathrm{~L} 2 / \mathrm{S} 2$ & $\mathrm{SAB}(\mathrm{r}) \mathrm{bc}$ \\
\hline NGC 2681 & 085332.8 & +511850 & 13.3 & L1.9 & $\left(\mathrm{R}^{\prime}\right) \mathrm{SAB}(\mathrm{rs}) 0 / \mathrm{a}$ \\
\hline NGC 2768 & 091137.5 & +600215 & 23.7 & $\mathrm{~L} 2$ & E6: \\
\hline NGC 2832 & 091946.9 & +334459 & 91.6 & L2:: & $E+2:$ \\
\hline NGC 2841 & 092202.6 & +505835 & 12.0 & $\mathrm{~L} 2$ & $\mathrm{SA}(\mathrm{r}) \mathrm{b}:$ \\
\hline NGC 2985 & 095021.6 & +721644 & 22.4 & T1.9 & $\left(\mathrm{R}^{\prime}\right) \mathrm{SA}(\mathrm{rs}) \mathrm{ab}$ \\
\hline NGC 3166 & 101345.6 & +032532 & 22.0 & $\mathrm{~L} 2$ & $\mathrm{SAB}(\mathrm{rs}) 0 / \mathrm{a}$ \\
\hline NGC 3169 & 101415.0 & +032757 & 19.7 & L2 & $\mathrm{SA}(\mathrm{s}) \mathrm{a}$ pec \\
\hline NGC 3190 & 101805.8 & +214956 & 22.4 & $\mathrm{~L} 2$ & SA(s)a pec spin \\
\hline NGC 3226 & 102327.0 & +195354 & 23.4 & L1.9 & E2: pec \\
\hline NGC 3301 & 103655.8 & +215255 & 23.3 & L2 & $\left(\mathrm{R}^{\prime}\right) \mathrm{SB}(\mathrm{rs}) 0 / \mathrm{a}$ \\
\hline NGC 3368 (M96) & 104645.7 & +114912 & 8.1 & L2 & $\mathrm{SAB}(\mathrm{rs}) \mathrm{ab}$ \\
\hline NGC 3414 & 105116.2 & +275830 & 24.9 & L2 & S0 pec \\
\hline NGC 3433 & 105203.9 & +100854 & 39.5 & L2/T2:: & $\mathrm{SA}(\mathrm{s}) \mathrm{c}$ \\
\hline NGC 3507 & 110325.4 & +180812 & 19.8 & L2 & $\mathrm{SB}(\mathrm{s}) \mathrm{b}$ \\
\hline NGC 3607 & 111654.3 & +180310 & 19.9 & $\mathrm{~L} 2$ & $\mathrm{SA}(\mathrm{s}) 0:$ \\
\hline NGC 3623 (M 65) & 111855.9 & +130532 & 7.3 & L2: & $\mathrm{SAB}(\mathrm{rs}) \mathrm{a}$ \\
\hline NGC 3626 & 112003.8 & +182125 & 26.3 & L2: & (R)SA(rs)0+ \\
\hline NGC 3627 (M 66) & 112015.0 & +125930 & 6.6 & $\mathrm{~T} 2 / \mathrm{S} 2$ & $\mathrm{SAB}(\mathrm{s}) \mathrm{b}$ \\
\hline NGC 3628 & 112017.0 & +133522 & 7.7 & $\mathrm{~T} 2$ & SAb pec spin \\
\hline NGC 3646 & 112214.7 & +201231 & 56.8 & $\mathrm{H} / \mathrm{T} 2:$ & $\mathrm{SB}(\mathrm{s}) \mathrm{a}$ \\
\hline NGC 3675 & 112607.9 & +433510 & 12.8 & $\mathrm{~T} 2$ & $\mathrm{SA}(\mathrm{S}) \mathrm{b}$ \\
\hline NGC 3718 & 113235.3 & +530401 & 17.0 & L1.9 & SB(s)a pec \\
\hline NGC 3780 & 113922.3 & +561614 & 37.2 & $\mathrm{~L} 2::$ & $\mathrm{SA}(\mathrm{s}) \mathrm{c}:$ \\
\hline NGC $3884^{a}$ & 114612.2 & +202330 & 91.6 & L1.9 & $\mathrm{SA}(\mathrm{r}) 0 / \mathrm{a}$ \\
\hline NGC 3898 & 114915.2 & +560504 & 21.9 & $\mathrm{~T} 2$ & $\mathrm{SA}(\mathrm{s}) \mathrm{ab}$ \\
\hline NGC 3945 & 115313.6 & +604032 & 22.5 & $\mathrm{~L} 2$ & $\mathrm{SB}(\mathrm{rs}) 0+$ \\
\hline NGC 4013 & 115831.3 & +435649 & 17.0 & $\mathrm{~T} 2$ & $\mathrm{SAb}$ \\
\hline NGC 4036 & 120126.9 & +615344 & 24.6 & L1.9 & S0- \\
\hline NGC 4143 & 120936.1 & +423203 & 17.0 & L1.9 & $\mathrm{SAB}(\mathrm{s}) 0$ \\
\hline NGC 4203 & 121505.0 & +331150 & 9.7 & L1.9 & SAB0-: \\
\hline NGC 4293 & 122112.8 & +182258 & 17.0 & L2 & $(\mathrm{R}) \mathrm{SB}(\mathrm{s}) 0 / \mathrm{a}$ \\
\hline NGC 4321 (M 100) & 122254.9 & +154921 & 16.8 & $\mathrm{~T} 2$ & $\mathrm{SAB}(\mathrm{s}) \mathrm{bc}$ \\
\hline NGC 4414 & 122627.1 & +311324 & 9.7 & $\mathrm{~T} 2:$ & $\mathrm{SA}(\mathrm{rs}) \mathrm{c} ?$ \\
\hline NGC 4435 & 122740.5 & +130444 & 16.8 & $\mathrm{~T} 2 / \mathrm{H}:$ & $\mathrm{SB}(\mathrm{s}) 0$ \\
\hline NGC 4438 & 122745.6 & +130032 & 16.8 & L1.9 & $\mathrm{SA}(\mathrm{s}) 0 / \mathrm{a}$ \\
\hline NGC 4450 & 122829.5 & +170506 & 16.8 & L1.9 & $\mathrm{SA}(\mathrm{s}) \mathrm{ab}$ \\
\hline NGC 4457 & 122859.1 & +033414 & 17.4 & $\mathrm{~L} 2$ & $(\mathrm{R}) \mathrm{SAB}(\mathrm{s}) 0 / \mathrm{a}$ \\
\hline NGC 4548 (M91) & 123526.4 & +142947 & 16.8 & L2 & $\mathrm{SBb}(\mathrm{rs})$ \\
\hline NGC 4589 & 123725.0 & +741131 & 30.0 & L2 & E2 \\
\hline NGC 4636 & 124250.0 & +024117 & 17.0 & L1.9 & E0+ \\
\hline NGC 4736 (M94) & 125053.0 & +410714 & 4.3 & L2 & $(\mathrm{R}) \mathrm{SA}(\mathrm{r}) \mathrm{ab}$ \\
\hline
\end{tabular}


Table 1. continued.

\begin{tabular}{|c|c|c|c|c|c|}
\hline $\begin{array}{l}\text { Galaxy } \\
\text { (1) }\end{array}$ & $\begin{array}{c}\mathrm{RA}(\mathrm{J} 2000) \\
\left(\begin{array}{c}\mathrm{h} \mathrm{m} \mathrm{s}) \\
(2)\end{array}\right.\end{array}$ & $\begin{array}{c}\operatorname{Dec}(\mathrm{J} 2000) \\
\left({ }^{\prime \prime} \prime \prime\right) \\
(3)\end{array}$ & $\begin{array}{c}D \\
(\mathrm{Mpc}) \\
(4)\end{array}$ & $\begin{array}{c}\text { Spectral type } \\
\text { (5) }\end{array}$ & $\begin{array}{l}\text { Hubble type } \\
\text { (6) }\end{array}$ \\
\hline NGC 4750 & 125007.1 & +725230 & 26.1 & L1.9 & (R)SA(rs)ab \\
\hline NGC 4772 & 125329.0 & +021002 & 16.3 & L1.9 & $\mathrm{SA}(\mathrm{s}) \mathrm{a}$ \\
\hline NGC 4826 (M 64) & 125643.7 & +214052 & 4.1 & $\mathrm{~T} 2$ & (R)SA(rs)ab \\
\hline NGC $5077^{b}$ & 131931.6 & -123926 & 40.6 & L1.9 & E3+ \\
\hline NGC 5297 & 134623.7 & +435220 & 37.8 & $\mathrm{~L} 2$ & SAB(s)c: spin \\
\hline NGC 5322 & 134915.2 & +601126 & 31.6 & $\mathrm{~L} 2::$ & E3+ \\
\hline NGC 5353 & 135326.7 & +401659 & 37.8 & L2/T2: & SA0 spin \\
\hline NGC 5363 & 135607.1 & +051520 & 22.4 & L2 & IA0? \\
\hline NGC 5371 & 135539.9 & +402743 & 37.8 & L2 & $\mathrm{SAB}(\mathrm{rs}) \mathrm{bc}$ \\
\hline NGC 5377 & 135616.6 & +471408 & 31.0 & $\mathrm{~L} 2$ & (R)SB(s)a \\
\hline NGC 5448 & 140249.7 & +491021 & 32.6 & L2 & (R)SAB(r)a \\
\hline NGC 5678 & 143205.8 & +575517 & 35.6 & $\mathrm{~T} 2$ & $\mathrm{SAB}(\mathrm{rs}) \mathrm{b}$ \\
\hline NGC 5813 & 150111.2 & +014208 & 28.5 & L2: & $\mathrm{E} 1+$ \\
\hline NGC 5838 & 150526.2 & +020558 & 28.5 & $\mathrm{~T} 2::$ & SA0- \\
\hline NGC 6340 & 171024.9 & +721816 & 22.0 & $\mathrm{~L} 2$ & $\mathrm{SA}(\mathrm{s}) 0 / \mathrm{a}$ \\
\hline NGC 6501 & 175603.7 & +182223 & 39.6 & L2:: & SA0+: \\
\hline NGC 6702 & 184657.6 & +454220 & 62.8 & L2:: & E: \\
\hline NGC 6703 & 184718.8 & +453302 & 35.9 & L2:: & SA0- \\
\hline
\end{tabular}

${ }^{a}$ Does not fulfill the magnitude criterion for the Palomar sample.

${ }^{b}$ Does not fulfill declination criterion for the Palomar sample.

P. Barthel, private communication 2005) and a VLBA/N05 detection of $7.3 \mathrm{mJy}$ Comparison of our MERLIN measurement with that of VLA/N05 yields an $\alpha \sim 0.0$.

NGC 3169: there is a NVSS (87.0 mJy) and FIRST $(16.4 \mathrm{mJy})$ radio detection; the flux density values show resolution effects. VLA/N00 (see also VLA/05) has detected a $6.8 \mathrm{mJy}$ source and FOO (see also VLBA/N05) detect $6.2 \mathrm{mJy}$; the source is compact on milliarcsecond scales. Using the VLA/N00 and F00 (see also VLA/N05 and VLBA/N05) values implies a flat spectral index for this radio source $(\alpha \sim 0.0)$. However, comparison of these values with our MERLIN measurement suggests the source could be radio variable.

NGC 3226: there is no NVSS value for this source, most likely because it is spatially confused with NGC 3227 . However, there is a 4.5 mJy FIRST detection. VLA/N00 (see also VLA/N05) and F00 (see also VLBA/N05) detect a 5.4 and $3.5 \mathrm{mJy}$ radio source, respectively, showing the source to be compact on milliarcsecond scales. From these last values the source appears to have a flat/inverted spectrum $(\alpha \gtrsim-0.4)$. The F00 measurement is consistent with our MERLIN value; the source does not appear variable at $5 \mathrm{GHz}$.

NGC 3414: the NVSS (4.4 mJy) and FIRST (4.0 mJy) flux densities show that this source is compact on arcsecond scales. Our MERLIN combined with VLA/N05 (2.4 mJy) yields an $\alpha \sim 0.0$.

NGC 3718: there is a NVSS (14.9 mJy) and FIRST (10.6 mJy) radio detection, whose flux density values show the source to be compact on arcsecond scales. There is also a VLA/N02 and VLBA/N02 (10.8 and 5.3 mJy detection, respectively (see also VLA/N05 and VLBA/N05). Comparison of these last values with our MERLIN measurement shows that
Table 2. Map parameters of the MERLIN-detected sources. Column 1: source name; Col. 2: spectral class from Ho et al. (1997a); Col. 3: restoring beam; Col. 4: position angle of the beam; Col. 5: rms noise level of the image.

\begin{tabular}{|c|c|c|c|c|}
\hline $\begin{array}{l}\text { Galaxy } \\
\text { (1) }\end{array}$ & $\begin{array}{c}\text { Spectral } \\
\text { Class } \\
(2)\end{array}$ & $\begin{array}{c}\text { Beamsize } \\
\left(\operatorname{mas}^{2}\right) \\
(3)\end{array}$ & $\begin{array}{l}\text { PA } \\
\text { (deg) } \\
(4)\end{array}$ & $\begin{array}{c}\mathrm{rms} \\
\text { (mJy/beam) } \\
(5)\end{array}$ \\
\hline N2768 & L2 & $130 \times 68$ & -7.07 & 0.10 \\
\hline N 3169 & $\mathrm{~L} 2$ & $178 \times 56$ & -45.27 & 0.09 \\
\hline N 3226 & L1.9 & $153 \times 48$ & -51.23 & 0.07 \\
\hline N3414 & L2 & $153 \times 49$ & -49.53 & 0.06 \\
\hline N 3718 & L1.9 & $161 \times 130$ & +54.66 & 0.06 \\
\hline $\mathrm{N} 3884^{a}$ & L1.9 & $86 \times 58$ & -79.10 & 0.05 \\
\hline N 4143 & L1.9 & $71 \times 41$ & +50.37 & 0.06 \\
\hline N 4203 & L1.9 & $126 \times 47$ & -28.85 & 0.06 \\
\hline N 4293 & $\mathrm{~L} 2$ & $159 \times 55$ & -18.22 & 0.06 \\
\hline N 4321 & $\mathrm{~T} 2$ & $174 \times 55$ & -15.41 & 0.06 \\
\hline N 4450 & L1.9 & $177 \times 57$ & -14.73 & 0.06 \\
\hline N $5077^{a}$ & L1.9 & $145 \times 47$ & +12.02 & 0.08 \\
\hline $\mathrm{N} 5297^{a}$ & $\mathrm{~L} 2$ & $71 \times 42$ & +29.53 & 0.06 \\
\hline N 5353 & L2 & $73 \times 44$ & +23.66 & 0.06 \\
\hline N 5363 & L2/T2: & $100 \times 46$ & +24.83 & 0.11 \\
\hline
\end{tabular}

${ }^{a}$ New subarcsecond-scale radio detection.

the source may suffer from resolution effects and/or can be radio variable $(\alpha \gtrsim-0.6)$.

NGC 3884: this source has a NVSS (14.0 mJy) and a FIRST (8.9 mJy) radio detection, showing some resolution effects. Taking into consideration the resolution mismatch, comparison of the FIRST and our MERLIN detection shows that the source may be resolved and/or radio variable $(\alpha<1.3)$. Because it 


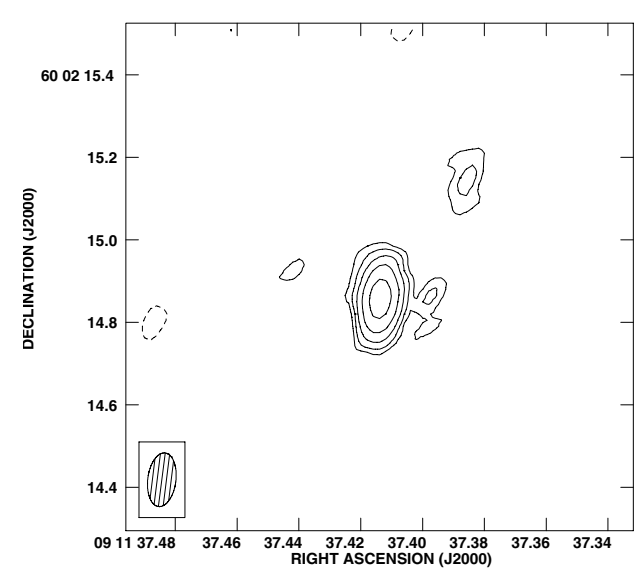

(a)

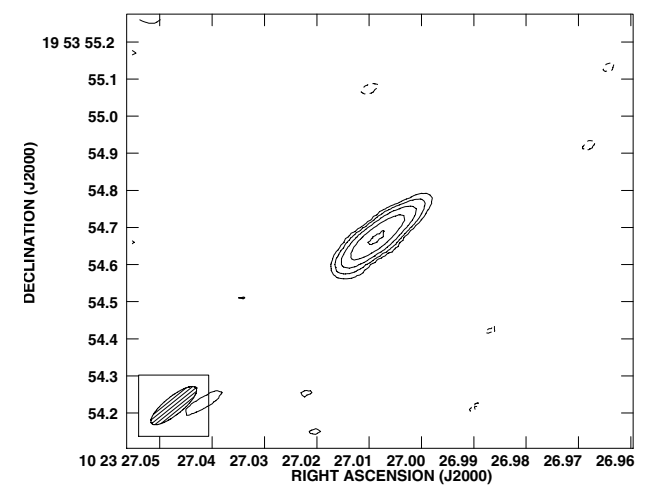

(c)

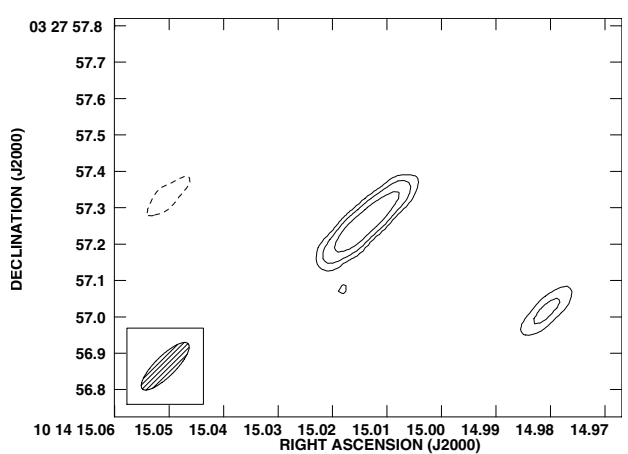

(b)

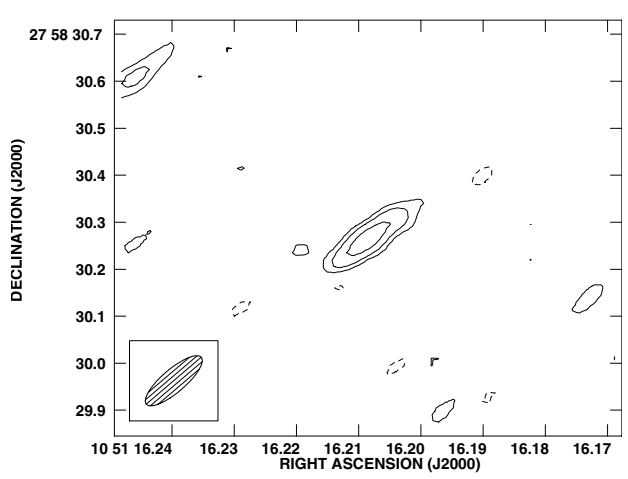

(d)

Fig. 1. Radio emission contours of the MERLIN-detected sources. Contour levels are rms $\times(-3,3,6,12,24,48,96,192)$, (see Table 2). The size of the restoring beam is given in each image at the bottom lefthand corner (see Table 2). a) NGC 2768, b) NGC 3169 , c) NGC 3226 and d) NGC 3414 .

does not fulfill the magnitude criterion for the Palomar survey, this source is not included in the final sample.

NGC 4143: there is a NVSS (9.9 mJy) and FIRST (5.0 $\mathrm{mJy})$ radio detection, showing some signs of resolution. This source was also observed in VLBA/N02 (8.7 mJy; see also VLBA/N05) and twice in the VLA/N00 and VLA/N02 (3.3 mJy and $10.0 \mathrm{mJy}$; see also VLA/N05). Comparison of these values with our MERLIN measurement shows that the source must be radio variable.

$N G C$ 4203: this source has been extensively observed at radio frequencies. There is a NVSS (6.1 mJy), FIRST (6.9 mJy), VLA/N00 and VLA/N02 (9.5 and $9.0 \mathrm{mJy}$; see also VLA/N05), F00 (8.9 mJy; see also VLBA/N05), HU01 (11.2 mJy) and multifrequency AUH04 radio detections. The flux density values, including the MERLIN detection, show that the source is compact and has a flat spectrum $(\alpha \sim 0.1)$.

$N G C$ 4293: this source has a NVSS (18.5 mJy) and a FIRST (13.9 $\mathrm{mJy}$ ) radio detection, denoting that it is compact on arcsecond scales. The present MERLIN and VLA/N02 (1.4 mJy; see also VLA/N05) measurements show that most of the source has been resolved and possesses a flat spectrum $(\alpha \lesssim 0.2)$. The weak amorphous nature of the MERLIN emission, associated with the flat spectrum, suggests the radio emission to be mainly thermal.
NGC 4321, M 100: there are several radio frequency measurements for this source (see source discussion in Filho et al. 2000). NVSS has detected $85.9 \mathrm{mJy}$ and FIRST a $37.4 \mathrm{mJy}$ source. The position of the MERLIN radio source is coincident with the optical nucleus of the galaxy as given by NED and with component A3 in Collison et al. (1994), the strongest component in their multiple radio structure map. The radio measurements clearly show the source has been resolved. Comparison of the Collison et al. (1994) values and our MERLIN detection, shows that the source is not variable at $5 \mathrm{GHz}$ and appears to have a fairly steep radio spectrum $(\alpha<1.6)$.

NGC 4450: comparison of the NVSS (9.4 mJy), FIRST (6.7 mJy), HU01 (6.5 mJy), VLA/N02 (2.7 mJy; see also VLA/N05), multifrequency AUH04 and MERLIN measurements show that the source is compact on arcsecond scales but has been partially resolved on subarcsecond scales. The AUH04 multifrequency data yield a spectral index of $\alpha \lesssim 0.3$.

NGC 4589: NVSS has detected a $36.8 \mathrm{mJy}$ radio source, VLA/N05 data yield $11.9 \mathrm{mJy}$ and VLBA/N05 data yield $11.5 \mathrm{mJy}$; the source is compact on subparsec scales. Our MERLIN data suffer from severe phase variations.

NGC 5077: the NVSS has detected a 156.7 mJy radio source. Our MERLIN map shows two very strong radio sources (A and B) in the nuclear region of this galaxy. The NVSS flux 


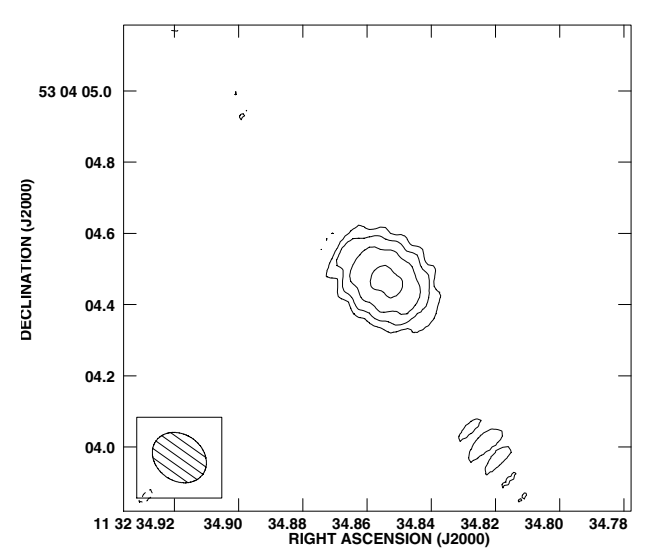

(a)

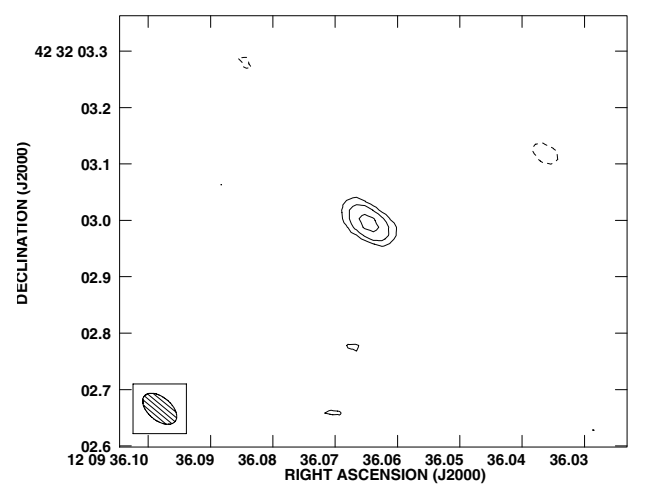

(c)

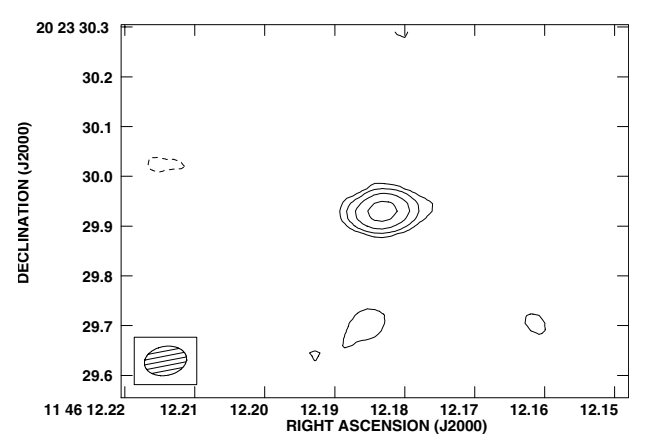

(b)

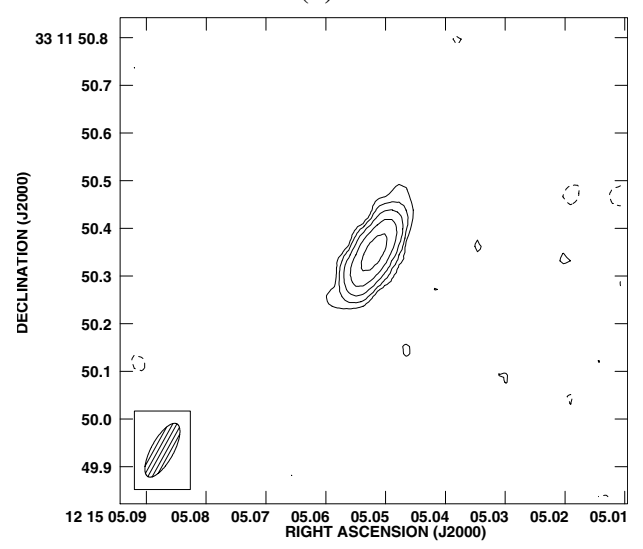

(d)

Fig. 2. As in Fig. 1. a) NGC 3718, b) NGC 3884, c) NGC 4143 and d) NGC 4203.

density appears to be the sum of the MERLIN flux of these two sources, denoting that there is little extended flux on arcsecond scales. While the APM and NED identify these radio sources with the nucleus of NGC 5077, it is difficult to constrain the origin of the radio emission without spectral information. Because it does not fulfill the declination criterion for the Palomar survey, this source is not included in the final sample.

NGC 5297: NVSS has detected a $23.0 \mathrm{mJy}$ radio source. FIRST has failed to detect this source most likely due to resolution. The MERLIN observations have resolved out a large part of the radio emission.

NGC 5353: this source has a NVSS (39.0 mJy) and FIRST (38.3 mJy) radio detection, denoting that the source is compact on arcsecond scales. There is also a VLBA/N05 (21.6 mJy) and VLA/N05 (18.7 mJy) detection. The MERLIN and VLA/N05 measurements yield an $\alpha \sim 0.0$.

NGC 5363: the FIRST (141.8 mJy) and NVSS (160.3 mJy) flux densities show that this source is compact on arcsecond scales. There is also a VLA/N05 and VLBA/N05 measurement of 40.7 and $39.6 \mathrm{mJy}$, respectively. The MERLIN observations have, however, resolved part of the radio emission into two components - A and B, with a total flux density of $\sim 25 \mathrm{mJy}$. Consultation of NED and the APM provides no other optical identification for these sources except sources belonging to the nuclear region of NGC 5363. It is possible that this source has a double nucleus.

\section{Background Sources}

$N G C$ 3627: as given by NED, we may have detected the

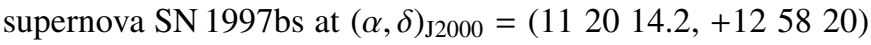
within less than $20^{\prime \prime}$ of the radio position measured in the map of this galaxy.

$N G C 4321$ : to the North, at the NED position $(\alpha, \delta)_{\mathrm{J} 2000}=$ (12 $2254.9,+154925$ ), there is emission coincident within less than $20^{\prime \prime}$ with the HII region (source 158), as documented in Hodge \& Kennicutt (1983).

NGC 4826: we do not detect the multiple source structure as found by Turner \& Ho (1994) because the individual sources either fall below our detection limit or fall outside the mapped region. We do, however, detect a radio source at the NED position $(\alpha, \delta)_{\mathrm{J} 2000}=(125643.66,+214103)$, which we identify, within less than $20^{\prime \prime}$, with a RSN (source 6) in Turner \& Ho (1994). Comparison of our and the Turner \& Ho (1994) fluxes suggest some variability.

Given the detection limit of the survey $-5 \sigma \sim$ $0.5 \mathrm{mJy}$ beam $^{-1}$ - the overall detection rate for the MERLIN observations is $27 \%$. We have excluded from this calculation the two sources which do not fulfill the magnitude/declination criteria, the HII and Seyfert galaxies (Table 1), eight sources with incorrect pointing relative to the $\mathrm{NED} /$ radio position and two sources with severe phase errors (see below). We can also estimate the fraction of MERLIN-detected sources $-58 \%$ - that are genuine AGN, due to the presence of broad $\mathrm{H} \alpha$ 


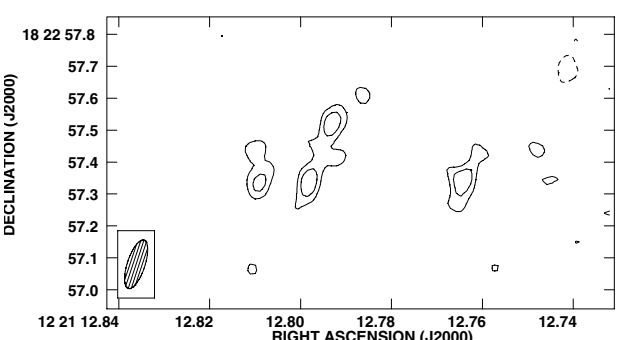

(a)

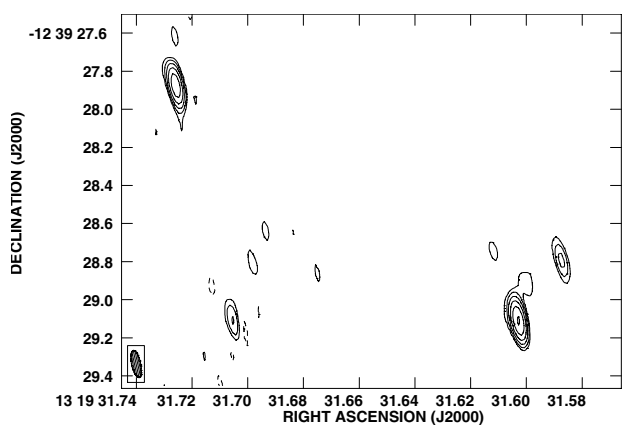

(c)

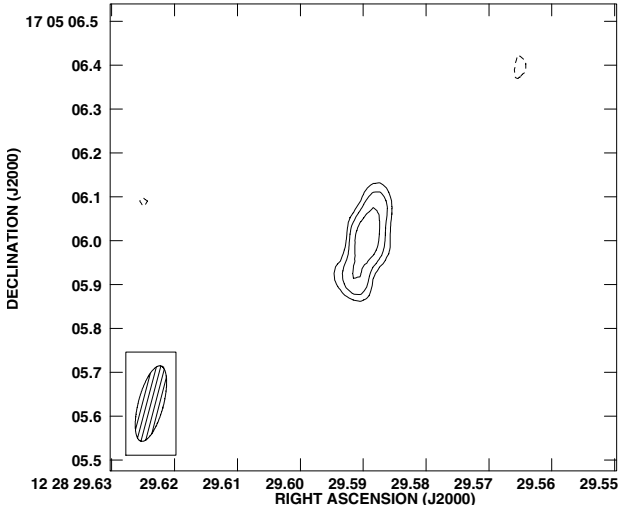

(b)

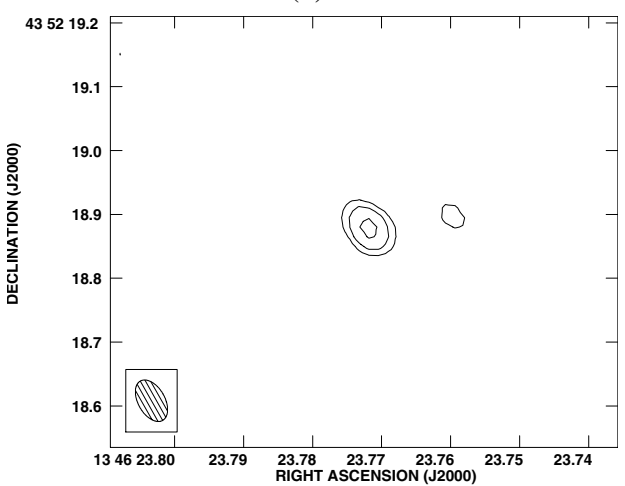

(d)

Fig. 3. As in Fig. 1. a) NGC 4293, b) NGC 4450, c) NGC 5077 and d) NGC 5297.

emission (type 1.9 sources; see also Sect. 5). Only $19 \%$ of the MERLIN-detected sources are type 2, which exhibit no broad emission lines. It is possible that type 2 sources are a mixed case, whereby only a small fraction of these are genuine AGN. However, various lines of evidence to be discussed below suggest that a significant fraction of these sources do indeed harbour a LLAGN. We will return to this issue in Sect. 5. The detection rate is $25 \%$ for the MERLIN-detected LINERs. This result is consistent with the $42 \%$ detection rate of LINERs in the VLA/N00 and VLA/N02 (see also VLA/N05) distancelimited sample, if we consider that $64 \%$ of our MERLIN LINER targets are farther than $20 \mathrm{Mpc}$.

We can also compare the MERLIN radio detections for the subset of sources (LINERs and composite galaxies) overlapping with the galaxy samples VLA/N00, VLA/N02 (see also VLA/N05), F00 (see also VLBA/05), HU01, and Filho et al. (2004). NGC 2678, NGC 2841, NGC 3169, NGC 3190, NGC 3226, NGC 3414, NGC 3607, NGC 3627, NGC 3628, NGC 3718, NGC 3780, NGC 3945, NGC 4143, NGC 4203, NGC 4293, NGC 4450, NGC 4548, NGC 4589, NGC 4636, NGC 4736, NGC 4772, NGC 5233, NGC 5353, NGC 5363, NGC 5377, NGC 5813 and NGC 5838, have been detected with the VLA and/or VLBA (VLA/N00; VLA/N02; VLA/N05; F00; VLBA/N05). NGC 777 (HU01) and NGC 5838 (Filho et al. 2004) have been detected with the VLA. Several sources (NGC 3190, NGC 3607, NGC 3627, NGC 3780, NGC 4548, NGC 4736 and NGC 4772) went undetected with MERLIN due to mispointing relative to the documented radio core.

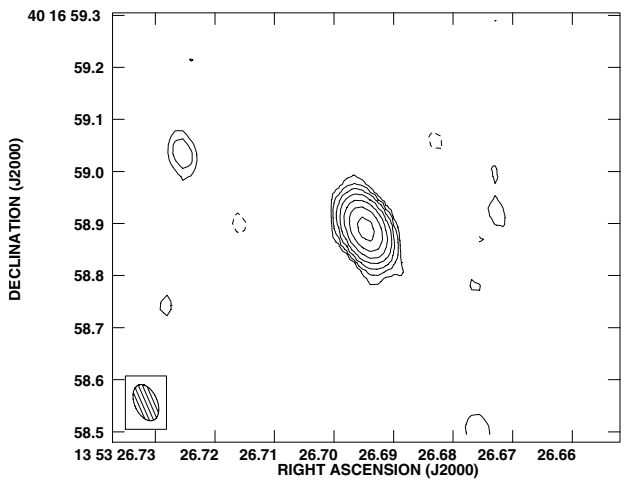

(a)

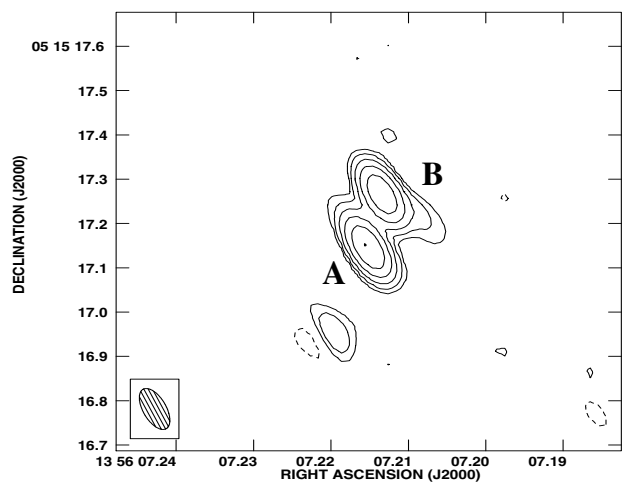

(b)

Fig. 4. As in Fig. 1. a) NGC 5353 and b) NGC 5363. 


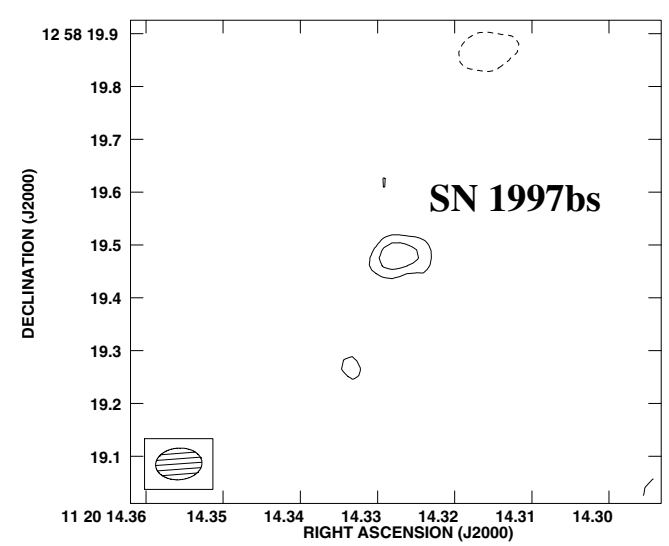

(a)

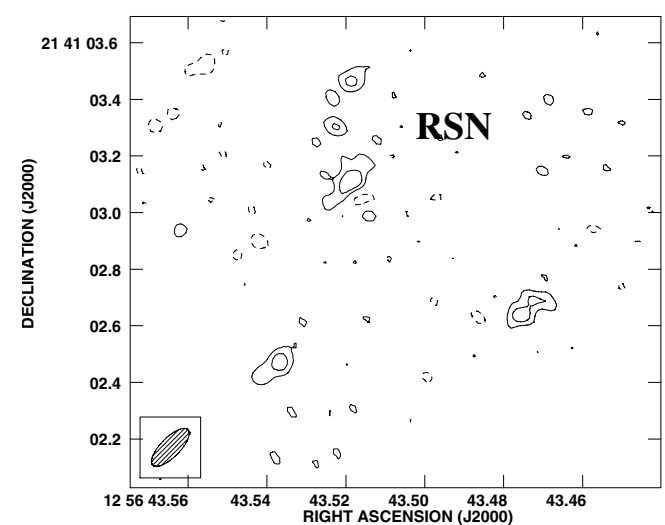

(b)

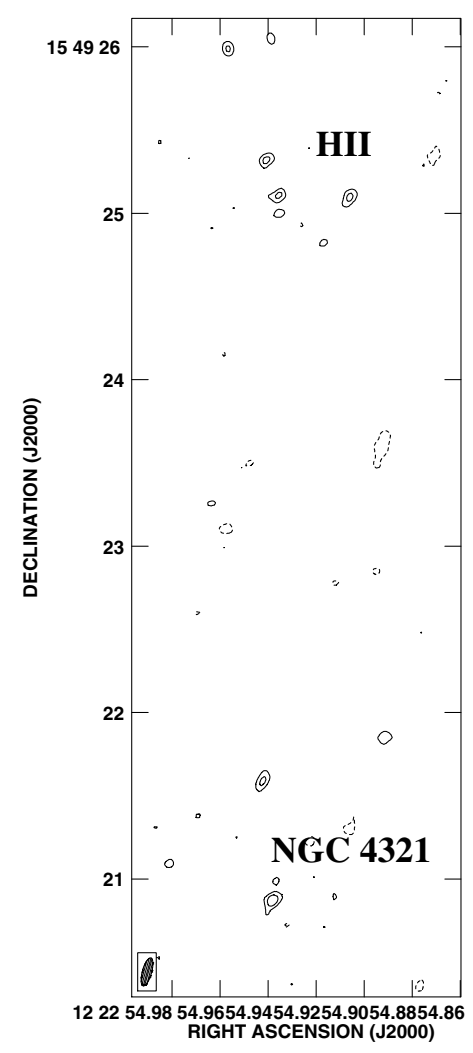

(c)

Fig. 5. MERLIN background detections (plus NGC 4321). Contours as in Fig. 1. a) Source positionally coincident with SN 1997bs in NGC 3627, b) source positionally coincident with a radio supernova (RSN) in NGC 4826 (source number 6; Turner \& Ho 1994) and c) source positionally coincident with an HII region (HII region number 158; Hodge \& Kennicutt 1983) in NGC 4321, whose central radio emission can be seen to the South.

One (NGC 4589) perhaps two (NGC 5322; see also Feretti et al. 1984) sources suffer from severe phase erros. As for NGC 2841, NGC 3628, NGC 3945, NGC 4636, NGC 5377 , NGC 5813 (VLA/N002; VLA/N02; VLA/N05), NGC 777 (HU01) and NGC 5838 (Filho et al. 2004; VLA/N05) which have peak flux densities below $2 \mathrm{mJy}$, it is likely our MERLIN observations either resolved out the emission or simply do not have enough signal-to-noise ratio. We note that the Lovell Telescope (Jodrell Bank), the most sensitive telescope of the MERLIN array, was offline during our observations. Of the remaining sources we have detected NGC 2768, NGC 3169,
NGC 3226, NGC 3718, NGC 4143, NGC 4203, NGC 4293, NGC 4450, NGC 5353, and NGC 5363 (Tables 2 and 3).

\section{Local radio luminosity function}

In order to construct a representative AGN radio luminosity function (RLF) of the local Universe, we have used the emission-line (excluding HII) sources in the Palomar sample. All of these sources have now been observed at 2 '.5 resolution or better, when we include the present MERLIN observations. We shall refer to the 196 LINERs, composite sources and 
Table 3. The $5 \mathrm{GHz}$ radio parameters of the MERLIN-detected sources. Column 1: source name; Col. 2: $5 \mathrm{GHz}$ peak radio flux density; Cols. 3 and 4: $5 \mathrm{GHz}$ radio position; Col. 5: integrated $5 \mathrm{GHz}$ flux density; Col. 6: brightness temperature.

\begin{tabular}{|c|c|c|c|c|c|}
\hline $\begin{array}{l}\text { Galaxy } \\
\text { (1) }\end{array}$ & $\begin{array}{c}F_{\text {peak }} \\
(\mathrm{mJy}) \\
(2)\end{array}$ & $\begin{array}{c}\mathrm{RA}(\mathrm{J} 2000) \\
\left(\begin{array}{c}\mathrm{h} \mathrm{m} \mathrm{s} \\
)\end{array}\right) \\
(3)\end{array}$ & 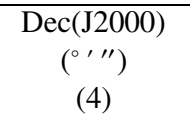 & $\begin{array}{c}F_{\text {int }} \\
(\mathrm{mJy}) \\
(5)\end{array}$ & $\begin{array}{c}T_{\mathrm{B}} \\
\left(\times 10^{4} \mathrm{~K}\right) \\
(6)\end{array}$ \\
\hline NGC 2768 & 7.06 & 091137.414 & +600214.86 & 7.22 & 3.9 \\
\hline NGC 3169 & 2.23 & 101415.014 & +03 2757.26 & 2.96 & 1.1 \\
\hline NGC 3226 & 3.50 & 102327.008 & +195354.67 & 3.88 & 2.3 \\
\hline NGC 3414 & 1.05 & 105116.208 & +275830.27 & 1.13 & 0.7 \\
\hline NGC 3718 & 1.65 & 113234.854 & +530404.47 & 2.07 & 0.4 \\
\hline NGC 3884 & 1.74 & 114612.183 & +202329.93 & 1.75 & 1.7 \\
\hline NGC 4143 & 0.97 & 120936.065 & +423203.00 & 1.00 & 1.6 \\
\hline NGC 4203 & 4.30 & 121505.052 & +331150.35 & 4.86 & 3.6 \\
\hline NGC 4293 & 0.54 & 122112.797 & +182257.41 & 1.81 & 0.3 \\
\hline NGC 4321 & 0.50 & 122254.938 & +154920.87 & 0.52 & 0.3 \\
\hline NGC 4450 & 1.21 & 122829.590 & +170506.00 & 1.23 & 0.6 \\
\hline NGC 5077A & 65.86 & 131931.726 & -123927.88 & 67.37 & 47.3 \\
\hline NGC 5077B & 85.03 & 131931.603 & -123929.11 & 86.66 & 61.1 \\
\hline NGC 5297 & 0.91 & 134623.772 & +435218.88 & 0.95 & 1.5 \\
\hline NGC 5353 & 16.21 & 135326.695 & +401658.89 & 17.42 & 24.7 \\
\hline NGC 5363A & 10.93 & 135607.215 & +051517.15 & 11.92 & 11.6 \\
\hline NGC 5363B & 10.95 & 135607.213 & +051517.26 & 11.28 & 11.7 \\
\hline
\end{tabular}

Table 4. Bibliographical radio data for the MERLIN-detected sources; Col. 1: survey; Col. 2: observing frequency; Col. 3: resolution; Col. 4: instrument; Col. 5: reference.

\begin{tabular}{lcccc}
\hline \hline $\begin{array}{l}\text { Survey } \\
(1)\end{array}$ & $\begin{array}{c}v \\
(\mathrm{GHz}) \\
(2)\end{array}$ & $\begin{array}{c}\text { Res. } \\
(\mathrm{mas}) \\
(3)\end{array}$ & $\begin{array}{c}\text { Instr. } \\
(4)\end{array}$ & $\begin{array}{c}\text { Ref. } \\
(5)\end{array}$ \\
\hline NVSS & 1.4 & 45000 & VLA & 1 \\
\hline FIRST & 1.4 & 5000 & VLA & 2 \\
\hline VLA/N00 & 15 & 150 & VLA & 3 \\
VLA/N02 & 15 & 150 & VLA & 4 \\
VLA/N05 & 15 & 150 & VLA & 5 \\
\hline VLBA/N02 & 5 & 2 & VLBA & 4 \\
VLBA/N05 & 5 & 2 & VLBA & 5 \\
F00 & 5 & 2 & VLBA & 6 \\
\hline HU01 & 5.0 & 1000 & VLA & 7 \\
\hline AUH04 & 1.7 & 10 & VLBA & 8 \\
& 2.3 & 6 & & \\
& 5.0 & 2 & & \\
& 8.4 & 1.6 & & \\
& 15.4 & 1.2 & & \\
& 22.2 & 0.8 & & \\
\hline
\end{tabular}

REFERENCES - (1) Condon et al. (1998); (2) Becker et al. (1995); (3) Nagar et al. (2000); (4) Nagar et al. (2002); (5) Nagar et al. (2005) and references therein; (6) Falcke et al. (2000); (7) Ho \& Ulvestad (2001); (8) Anderson et al. (2004).

Seyferts which satisfy both the magnitude $\left(B_{\mathrm{T}}<12.5 \mathrm{mag}\right)$ and declination criteria $(\delta>0 \mathrm{deg})$ of the Palomar survey as the "LTS sources" for brevity (LTS meaning LINER-TransitionSeyfert); these sources constitute the present sample.

Ideally, we would like to have a homogeneous set of radio observations. But lacking such a survey, we have assembled in
Table 5. Radio data used in the construction of the LTS source RLF. Column 1: spectral class or galaxy; Col. 2: number of relevant sources surveyed. In parentheses are the number of radio-detected sources. Please note that some sources may be in multiple radio surveys; Col. 3 : observing frequency; Col. 4: resolution; Col. 5: instrument; Col. 6: flux limit $(5 \sigma)$; Col. 7 : reference.

\begin{tabular}{lcccccc}
\hline \hline Class/ & & $v$ & Res. & & $\mathrm{F}_{\text {limit }}$ \\
Galaxy & $N$ & $(\mathrm{GHz})$ & $(\mathrm{mas})$ & Instr. & $(\mathrm{mJy})$ & Ref. \\
$(1)$ & $(2)$ & $(3)$ & $(4)$ & $(5)$ & $(6)$ & $(7)$ \\
\hline Seyfert & $52(37)$ & 5 & 1000 & VLA & 0.2 & 1 \\
\hline LINER & $45(19)$ & 15 & 150 & VLA & 1.0 & 2,3 \\
& $88(37)$ & 15 & 150 & VLA & 1.0 & 4 \\
& $52(14)$ & 5 & 100 & MERLIN & 0.5 & 5 \\
\hline Composite & $39(7)$ & 15 & 150 & VLA & 1.1 & 2,3 \\
& $62(9)$ & 15 & 150 & VLA & 1.0 & 4 \\
& $16(1)$ & 5 & 100 & MERLIN & 0.5 & 5 \\
& $35(26)$ & 8.4 & 2500 & VLA & 0.3 & 6,7 \\
NGC 660 & $\ldots$ & 8.4 & 200 & VLA & 0.03 & 8 \\
NGC 7331 & $\ldots$ & 5 & 2000 & VLA & 0.1 & 9 \\
\hline
\end{tabular}

REFERENCES - (1) Ho \& Ulvestad (2001); (2) Nagar et al. (2002); (3) Nagar et al. (2000); (4) Nagar et al. (2005) and references therein; (5) this paper; (6) Filho et al. (2002a); (7) Filho et al. (2000); (8) data kindly provided by J. Ulvestad; (9) Cowan et al. (1994).

Table 5 a list of radio measurements used to derive the RLF for the LTS sample sources. Radio observations at different frequencies have been converted to $5 \mathrm{GHz}$ assuming a spectral index of 0.7 and also corrected for different cosmologies if necessary. When multiple observations of the same galaxy were available, by order of preference we choose HU01 for Seyferts, the radio observations in Filho et al. (2000, 2002a) and VLA/N00, VLA/N02 (see also VLA/N05) for composite galaxies, VLA/N00, VLA/N02 (see also VLA/N05) and present MERLIN observations for LINERs.

We caution that the radio-detection rate will depend strongly on observing frequency, resolution and sensitivity. At higher frequencies, many sources may escape detection because spectral indices are not always flat and instrumental sensitivities will also be lower. Furthermore, the source may suffer resolution effects. With this in mind, the VLA/N00, VLA/N02 samples (see also VLA/N05), Filho et al. (2000, 2002a) and the present MERLIN observations provide only lower limits to the radio-detection rate in LTS sources.

Galaxies are considered detected if their radio flux density is above $5 \sigma$, where $\sigma$ is the typical noise associated with the respective survey (Table 5). 82\%, 43\%, and $49 \%$ of the Seyferts, LINERs, and composite galaxies were detected in the radio at 2 .'5 resolution or less. This is equivalent to an overall radio-detection rate of $54 \%$ in all LTS sources or $22 \%$ of all bright nearby galaxies (Palomar sources). For type 1 and 2 LTS sources, the radio detections are $89 \%$ and $46 \%$, respectively. Of the type 1 radio-detected sources (which must be genuine AGN), 59\% are classified as Seyferts and 35\% are LINERs. Both broad-lined composite galaxies (type 1.9 NGC 1161 and NGC 2985) were detected. $100 \%$ of type 1 Seyferts and $67 \%$ of type 1 LINERs were detected. $46 \%, 68 \%$, and $94 \%$ of the 
type 2 radio-detected sources are Seyferts, LINERs, and composite galaxies, respectively.

We can also estimate the fraction of these radio-detected LTS sources that are likely to be genuine AGN. In the absence of unambiguous optical spectral signatures of AGN activity (e.g., broad emission lines), the radio regime provides an alternative and complementary diagnostic. Although not a necessary condition for the presence of an AGN, a compact, flat-spectrum radio core is indicative of synchrotron selfabsorption, which is associated with jet emission from AGN. However, there are several caveats. First, ground-based optical spectra and VLBI-resolution radio images typically sample very different spatial, and presumably temporal, scales. Thus, optical and radio signatures of AGN activity need not occur concurrently. Second, because the mechanism of jet formation is still uncertain, radio emission cannot be regarded as an inevitable by-product of AGN activity. Finally, empirical evidence suggests that there is a radio flux density threshold of $2 \mathrm{mJy}$ below which the sources become difficult to detect at milliarcsecond-scale resolution using current facilities (F00; VLBA/N05; Filho et al. 2004). Known sources with submilliJansky radio cores and/or hard X-ray detections (e.g., NGC 660 and NGC 7331; see Filho et al. 2002a, 2004), which are likely to be genuine AGN, or sources that are highly radio variable can be missed by these relatively shallow, milliarcsecondscale resolution observations. We therefore caution that our estimate based on radio detection is a lower limit to the true AGN fraction in LTS sources. For the sake of homogeneity, we will restrict ourselves to observations of $\lesssim 1^{\prime \prime}$ resolution. With the above caveats in mind, we conclude that at least $80 \%$ of the Seyferts (HU01), $40 \%$ of the LINERs (the present MERLIN observations; VLA/NO0; VLA/N02; VLA/N05) and $20 \%$ of the composite sources (the present MERLIN observations; VLA/N00; VLA/N02; VLA/N05) are likely AGN. Based on the presence of compact radio emission, the total fraction of LTS sources and bright nearby galaxies harbouring AGN is therefore $\sim 50 \%$ and $\sim 20 \%$, respectively.

According to classical AGN unification schemes (Antonucci 1993), type 2 objects are simply type 1 AGN seen edge-on, whereby the molecular torus blocks the direct view of the broad-line region (BLR). However, in the case of LLAGN it is not entirely clear that unification schemes are readily applicable. There is growing evidence that not all low luminosity Seyferts, LINERs and composite sources possess a BLR and when they do the BLR is weak (Barth et al. 1999); there are only twelve sources in the Palomar sample (Ho et al. 1997a) classified as type 1.0-1.8 and all are Seyferts. We can then conservatively argue that all type 1 sources, which exhibit broad-line emission in their spectra, are genuine AGN. Therefore, based solely on the presence of broad-line emission, we can estimate that at least $20 \%$ of the LTS sources and $10 \%$ of all bright nearby galaxies harbour an AGN.

Because the sample sources are nearby (median $D=$ $17 \mathrm{Mpc}$ ), we have considered a flat, Euclidean Universe with $q_{0}=0.5$ and $H_{0}=75 \mathrm{~km} \mathrm{~s}^{-1} \mathrm{Mpc}^{-1}$ for the subsequent calculations. The $V / V_{\max }$ method (Schmidt 1968) was applied in order to construct the RLF at $5 \mathrm{GHz}$. The main constraints arise from the magnitude limit of the Palomar survey which is taken to be
$B_{\text {limit }}=12.5 \mathrm{mag}$ (Ho et al. 1995) and the radio flux limit of the survey from where the radio luminosity of the LTS source was obtained (Table 5). Moreover, only galaxies with positive declination were observed, which restricts the survey area covered to $2 \pi$. The calculation of the RLF is then performed over equal bins in $\log$ of radio power $(0.4 \mathrm{dex})$. In each bin centered on the radio luminosity $\log L^{*}$, the space density (or differential LF) follows from the expression:

$\Phi\left(\log L^{*}\right)=\frac{4 \pi}{\Omega} \sum_{i=1}^{n\left(\log L^{*}\right)} \frac{1}{V_{\max (i)}}$,

where $n\left(\log L^{*}\right)$ is the number of galaxies in the bin with luminosities between $\log L^{*}-0.2$ and $\log L^{*}+0.2, \frac{4 \pi}{\Omega}$ is the fraction of the sky covered by the optical survey, and $V_{\max }$ is the maximum volume of the sphere in which the source could have been detected given both the magnitude and radio flux limits ( $\equiv \frac{4 \pi}{3} d_{\max (\mathrm{i})}{ }^{3}$ ), with the maximum volume being the smaller of the two. Statistical errors associated with the space densities were assigned assuming Poisson statistics. The total number of objects per unit volume brighter than $\log L^{*}\left(\Psi\left(\log L^{*}\right)\right.$; cumulative version of the RLF) is obtained by summing over all the space densities in bins with $\log L<\log L^{*}$ :

$\Psi\left(\log L^{*}\right)=\Sigma_{i=1}^{n\left(\log L<\log L^{*}\right)} \Phi\left(\log L^{*}\right)$.

\section{Discussion}

Inspection of Fig. 6a shows that space densities of LTS sources continue to rise with decreasing radio power, with some evidence of flattening below $10^{20} \mathrm{~W} \mathrm{~Hz}^{-1}$, partly due to incompleteness of the radio survey (see also discussion in Nagar et al. 2005). At all radio powers the space densities are clearly dominated by the LINER galaxies, in particular at the high radio power end $\left(>10^{22} \mathrm{~W} \mathrm{~Hz}^{-1}\right)$. Both Seyfert and LINER galaxies contribute to the steady rise in space densities. On the other hand, the space densities of composite sources appear relatively flat in the range $19<\log P_{5 \mathrm{GHz}}<21 \mathrm{~W} \mathrm{~Hz}^{-1}$.

Table 6 and Fig. 6 contain the derived RLF for the radiodetected LTS sample sources, together and separately for for Seyferts, LINERs, composite sources and then also for type 1 and type 2 objects (with and without broad lines, respectively). It is worth mentioning that, particularly on the low-power end, the RLF is irregular; this is most likely due to the density inhomogeneities in our local volume.

At all radio powers, the space densities in Fig. $6 \mathrm{~b}$ are dominated by the more numerous type 2 sources, although $56 \%$ of the type 2 sources were not detected on scales $<2$ '. 5 They do, however, span the same radio power range as type 1 sources and appear to turn over at a power of $10^{21} \mathrm{~W} \mathrm{~Hz}^{-1}$. Type 2 sources roughly mimic the composite source and Seyfert behaviour in its flattening below $\log P_{5 \mathrm{GHz}}=21 \mathrm{~W} \mathrm{~Hz}^{-1}$.

It is possible that type 2 or (equivalently composite sources and some LINERs) are a mixed case, whereby only a small fraction of these are genuine AGN. However, various lines of evidence, as stated above, suggest that we are underestimating the number of LLAGN in the LTS sample. Long integration X-ray and radio observations should prove useful in this regard to provide a complete survey with uniform sensitivity 


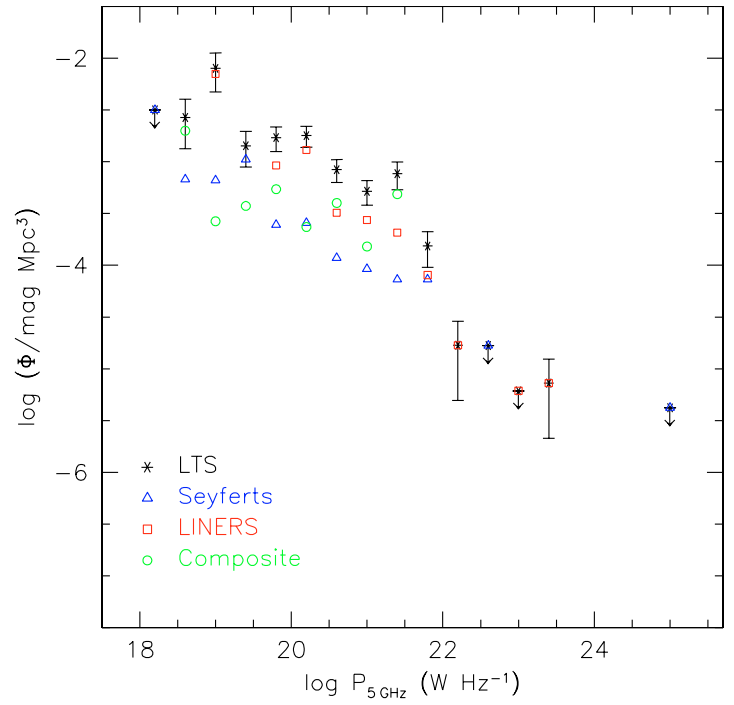

(a)

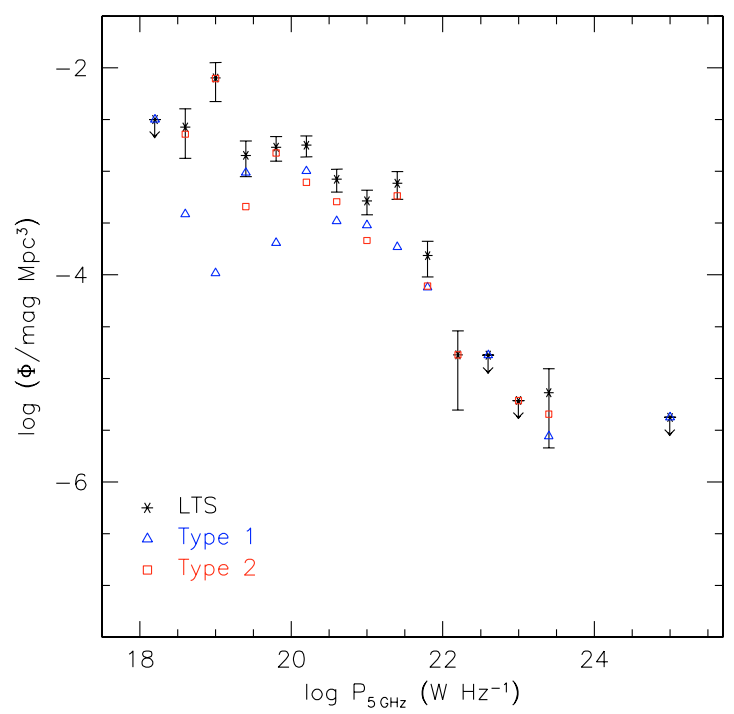

(b)

Fig. 6. The radio luminosity function for the radio-detected LTS sample sources: a) Seyferts, LINERs and composite galaxies and b) type 1 and type 2 sources. Downward arrows are for bins with only one galaxy. Errorbars are assigned assuming Poisson statistics.

and resolution. If there is a significant population of submillijansky LLAGN that we cannot detect with the present observations, then by including them in the RLF, we should expect higher space densities, in particular at the low-luminosity end of the RLF.

Furthermore, because we have compiled radio measurements from surveys with different resolutions, is it likely that in some cases we are overestimating radio flux densities. Many of these sources are known to suffer from resolution effects (Filho et al. 2000, 2002a; VLA/N05; VLBA/N05; Filho et al. 2004); the radio power of the underlying AGN may be an order of magnitude or so lower than given by the arcsec-scale observations. The overall effect would be to shift the RLF to lower radio luminosities.

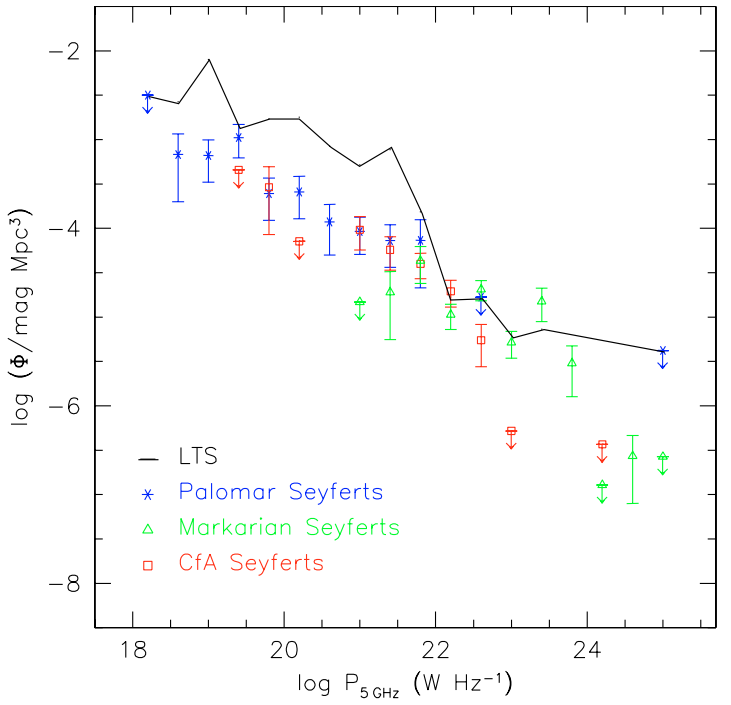

(a)

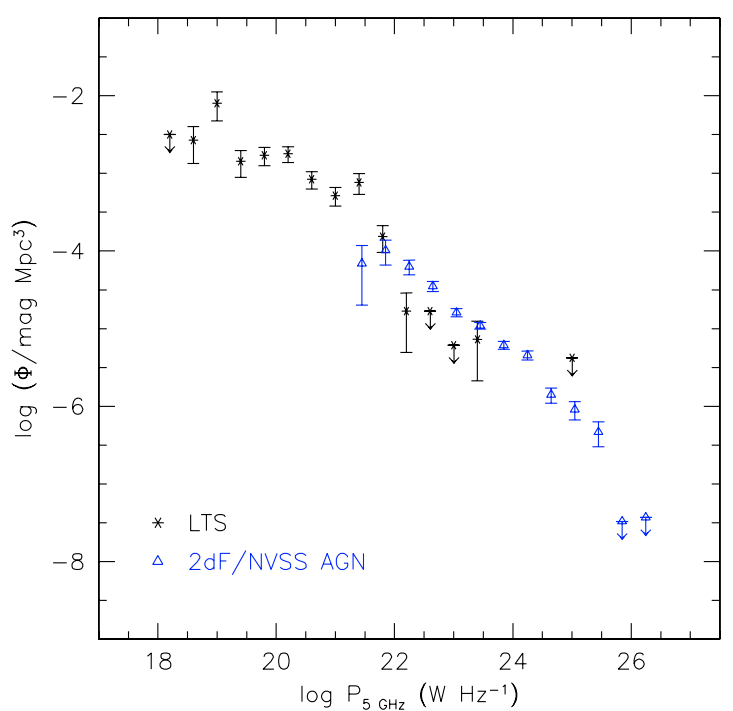

(b)

Fig. 7. The radio luminosity function for the radio-detected LTS sources compared to: a) the Palomar, CfA (Huchra \& Burg 1992; radio data from Kukula et al. 1995) and Markarian Seyferts (Meurs \& Wilson 1984) and b) compared to $2 \mathrm{dF} / \mathrm{NVSS}$ AGN (Sadler et al. 2002). Downward arrows are for bins with only one galaxy. Errorbars are assigned assuming Poisson statistics.

It is interesting to compare our results with available published surveys. In VLA/N05 the authors have derived a RLF for a distance-limited sample of Palomar sources. Comparison between our analysis and theirs shows that the results are in rough agreement, within the errors. In particular, the larger sample of the LTS sources presented in this study (our 106 versus their 68 radio-detected sources) allows us to confirm the low-power turnover seen in the RLF.

We have also derived a RLF for the Markarian (Meurs \& Ulvestad 1984) and CfA Seyferts (Huchra \& Burg 1992; 2 " resolution VLA radio data from Kukula et al. 1995), converting flux densities to $5 \mathrm{GHz}$ assuming $\alpha=0.7$ (Fig. 7a). We caution that the Markarian Seyferts have been observed 
Table 6. The local radio luminosity function. Column 1: binned radio power; Cols. 2, 5, 8: number of galaxies in the radio magnitude bin; Cols. 3, 6, 9: space density of galaxies and error, calculated assuming Poisson statistics; Cols. 4, 7, 10: cumulative number of galaxies.

\begin{tabular}{|c|c|c|c|c|c|c|c|c|c|}
\hline $\begin{array}{c}P \\
\left(\mathrm{~W} \mathrm{~Hz}^{-1}\right) \\
(1)\end{array}$ & $\begin{array}{l}N \\
\text { (2) }\end{array}$ & $\begin{array}{c}\log \Phi \\
\left(\mathrm{mag}^{-1} \mathrm{Mpc}^{-3}\right) \\
\text { (3) }\end{array}$ & $\begin{array}{c}\log \Psi \\
\left(\mathrm{Mpc}^{-3}\right) \\
(4)\end{array}$ & $\begin{array}{l}N \\
\text { (5) }\end{array}$ & $\begin{array}{c}\log \Phi \\
\left(\mathrm{mag}^{-1} \mathrm{Mpc}^{-3}\right) \\
(6)\end{array}$ & $\begin{array}{c}\log \Psi \\
\left(\mathrm{Mpc}^{-3}\right) \\
(7)\end{array}$ & $\begin{array}{c}N \\
(8)\end{array}$ & $\begin{array}{c}\log \Phi \\
\left(\mathrm{mag}^{-1} \mathrm{Mpc}^{-3}\right) \\
(9)\end{array}$ & $\begin{array}{c}\log \Psi \\
\left(\mathrm{Mpc}^{-3}\right) \\
(10)\end{array}$ \\
\hline & \multicolumn{3}{|c|}{ Seyferts } & \multicolumn{3}{|c|}{ LINERs } & \multicolumn{3}{|c|}{ Composite } \\
\hline 18.2 & 1 & $-2.50_{-1.00}^{+0.30}$ & -2.50 & $\ldots$ & $\ldots$ & $\ldots$ & $\ldots$ & $\ldots$ & $\ldots$ \\
\hline 18.6 & 2 & $-3.17_{-0.53}^{+0.23}$ & -2.41 & $\ldots$ & $\ldots$ & $\ldots$ & 2 & $-2.70_{-0.53}^{+0.23}$ & -2.70 \\
\hline 19.0 & 4 & $-3.18_{-0.30}^{+0.18}$ & -2.35 & 1 & $-2.15_{-1.00}^{+0.30}$ & -2.15 & 1 & $-3.58_{-1.00}^{+0.33}$ & -2.65 \\
\hline 19.4 & 6 & $-2.98_{-0.23}^{+0.15}$ & -2.25 & ... & $-2.15_{-1.00}^{+0.30}$ & -2.12 & 1 & $-3.43_{-1.00}^{+0.30}$ & -2.58 \\
\hline 19.8 & 4 & $-3.61_{-0.30}^{+0.18}$ & -2.24 & 3 & $-3.04_{-0.38}^{+0.20}$ & -2.10 & 7 & $-3.26_{-0.21}^{+0.14}$ & -2.50 \\
\hline 20.2 & 4 & $-3.59_{-0.30}^{+0.18}$ & -2.22 & 9 & $-2.89_{-0.18}^{+0.12}$ & -2.03 & 6 & $-3.63_{-0.23}^{+0.15}$ & -2.47 \\
\hline 20.6 & 3 & $-3.93_{-0.37}^{+0.20}$ & -2.21 & 5 & $-3.49_{-0.26}^{+0.16}$ & -2.02 & 8 & $-3.40_{-0.19}^{+0.13}$ & -2.42 \\
\hline 21.0 & 5 & $-4.04_{-0.26}^{+0.16}$ & -2.20 & 6 & $-3.56_{-0.23}^{+0.15}$ & -2.01 & 3 & $-3.82_{-0.37}^{+0.20}$ & -2.40 \\
\hline 21.4 & 4 & $-4.14_{-0.30}^{+0.18}$ & -2.20 & 3 & $-3.68_{-0.37}^{+0.20}$ & -2.00 & 4 & $-3.31_{-0.30}^{+0.18}$ & -2.35 \\
\hline 21.8 & 2 & $-4.14_{-0.53}^{+0.23}$ & -2.19 & 5 & $-4.09_{-0.28}^{+0.16}$ & -2.00 & $\ldots$ & $\ldots$ & $\ldots$ \\
\hline 22.2 & $\ldots$ & $\ldots$ & -2.19 & 2 & $-4.77_{-0.53}^{+0.23}$ & -2.00 & $\ldots$ & $\ldots$ & $\cdots$ \\
\hline 22.6 & 1 & $-4.78_{-1.00}^{+0.30}$ & -2.19 & $\ldots$ & $\ldots$ & -2.00 & $\ldots$ & $\ldots$ & $\ldots$ \\
\hline 23.0 & $\ldots$ & $\ldots$ & -2.19 & 1 & $-5.21_{-1.00}^{+0.30}$ & -2.00 & $\ldots$ & $\ldots$ & $\ldots$ \\
\hline 23.4 & $\ldots$ & $\ldots$ & -2.19 & 2 & $5.14_{-0.53}^{+0.23}$ & -2.00 & $\ldots$ & $\ldots$ & $\ldots$ \\
\hline 23.8 & $\ldots$ & $\ldots$ & -2.19 & $\ldots$ & $\ldots$ & $\ldots$ & $\ldots$ & $\ldots$ & $\ldots$ \\
\hline 24.2 & $\ldots$ & $\ldots$ & -2.19 & $\ldots$ & $\ldots$ & $\ldots$ & $\ldots$ & $\ldots$ & $\ldots$ \\
\hline 24.6 & $\ldots$ & $\ldots$ & -2.19 & $\ldots$ & $\ldots$ & $\ldots$ & $\ldots$ & $\ldots$ & $\ldots$ \\
\hline 25.0 & 1 & $-5.38_{-0.30}^{+1.00}$ & -2.19 & $\ldots$ & $\ldots$ & $\ldots$ & $\ldots$ & $\ldots$ & $\ldots$ \\
\hline \multirow[t]{2}{*}{ Total } & 37 & $\ldots$ & $\ldots$ & 32 & $\ldots$ & $\ldots$ & 37 & $\ldots$ & $\ldots$ \\
\hline & \multicolumn{3}{|c|}{ type 1} & \multicolumn{3}{|c|}{ type 2} & All & & \\
\hline 18.2 & 1 & $-2.50_{-1.00}^{+0.30}$ & -2.50 & $\ldots$ & $\ldots$ & $\ldots$ & 1 & $-2.50_{-1.00}^{+0.30}$ & -2.50 \\
\hline 18.6 & 1 & $-3.41_{-1.00}^{+0.30}$ & -2.45 & 3 & $-2.64_{-0.37}^{+0.20}$ & -2.64 & 4 & $-2.57_{-0.30}^{+0.18}$ & -2.23 \\
\hline 19.0 & 1 & $-3.98_{-1.00}^{+0.30}$ & -2.44 & 5 & $-2.10_{-0.26}^{+0.16}$ & -1.99 & 6 & $-2.10_{-0.23}^{+0.15}$ & -1.86 \\
\hline 19.4 & 3 & $-3.01_{-0.37}^{+0.20}$ & -2.33 & 4 & $-3.34_{-0.30}^{+0.18}$ & -1.97 & 7 & $-2.86_{-0.21}^{+0.14}$ & -1.82 \\
\hline 19.8 & 2 & $-3.69_{-0.53}^{+0.23}$ & -2.31 & 12 & $-2.82_{-0.15}^{+0.11}$ & -1.92 & 14 & $-2.77_{-0.14}^{+0.10}$ & -1.77 \\
\hline 20.2 & 8 & $-3.00_{-0.19}^{+0.13}$ & -2.23 & 11 & $-3.11_{-0.16}^{+0.11}$ & -1.89 & 19 & $-2.75_{-0.11}^{+0.09}$ & -1.73 \\
\hline 20.6 & 4 & $-3.48_{-0.30}^{+0.18}$ & -2.21 & 12 & $-3.30_{-0.15}^{+0.11}$ & -1.87 & 16 & $-3.08_{-0.13}^{+0.10}$ & -1.71 \\
\hline 21.0 & 7 & $-3.52_{-0.21}^{+0.14}$ & -2.19 & 7 & $-3.67_{-0.21}^{+0.14}$ & -1.87 & 14 & $-3.29_{-0.14}^{+0.10}$ & -1.70 \\
\hline 21.4 & 1 & $-3.73_{-1.00}^{+0.30}$ & -2.18 & 10 & $-3.24_{-0.18}^{+0.12}$ & -1.85 & 11 & $-3.11_{-0.16}^{+0.11}$ & -1.68 \\
\hline 21.8 & 3 & $-4.12_{-0.37}^{+0.20}$ & -2.17 & 4 & $-4.11_{-0.30}^{+0.18}$ & -1.84 & 7 & $-3.81_{-0.21}^{+0.14}$ & -1.68 \\
\hline 22.2 & $\ldots$ & $\ldots$ & -2.17 & 2 & $-4.77_{-0.53}^{+0.23}$ & -1.84 & 2 & $-4.77_{-0.53}^{+0.23}$ & -1.68 \\
\hline 22.6 & 1 & $-4.78_{-1.00}^{+0.30}$ & -2.17 & .. & $\cdots$ & -1.84 & 1 & $-4.78_{-1.00}^{+0.30}$ & -1.68 \\
\hline 23.0 & $\ldots$ & $\ldots$ & -2.17 & 1 & $-5.21_{-1.00}^{+0.30}$ & -1.84 & 1 & $-5.21_{-1.00}^{+0.30}$ & -1.68 \\
\hline 23.4 & 1 & $-5.56_{-1.00}^{+0.30}$ & -2.17 & 1 & $-5.35_{-1.00}^{+0.30}$ & -1.84 & 2 & $-5.14_{-0.53}^{+0.23}$ & -1.68 \\
\hline 23.8 & $\ldots$ & $\ldots$ & -2.17 & $\ldots$ & $\ldots$ & $\ldots$ & $\ldots$ & $\ldots$ & -1.68 \\
\hline 24.2 & $\ldots$ & $\ldots$ & -2.17 & $\ldots$ & $\ldots$ & $\ldots$ & $\ldots$ & $\ldots$ & -1.68 \\
\hline 24.6 & $\ldots$ & $\ldots$ & -2.17 & $\ldots$ & $\ldots$ & $\ldots$ & $\ldots$ & $\ldots$ & -1.68 \\
\hline 25.0 & 1 & $-5.38_{-0.30}^{+1.00}$ & -2.17 & .. & $\ldots$ & $\ldots$ & 1 & $-5.38_{-1.00}^{+0.30}$ & -1.68 \\
\hline Total & 34 & $\ldots$ & $\ldots$ & 72 & $\ldots$ & $\ldots$ & 106 & $\ldots$ & $\ldots$ \\
\hline
\end{tabular}

with Westerbork Radio Synthesis Telescope (WRST) and nuclear radio flux densities may be over-estimated. The RLFs are consistent with the Palomar Seyfert and RLF for powers above $10^{21} \mathrm{~W} \mathrm{~Hz}^{-1}$ (Fig. 7a). The rising of the LTS and Palomar Seyfert RLF towards lower powers demonstrates that the LTS sample contains fainter and more local sources than those in the Markarian and CfA samples. This result is in agreement with that found in VLA/N05 and in HU01, considering the difference in RLF frequency and binning.
Similarly, we can compare our RLF with the AGN sources in the 2dF Galaxy Redshift Survey of Sadler et al. (2002). Galaxies in this sample have been classified as AGN according to their spectral characteristics; they show either an absorption-line spectrum like that of a giant elliptical, an absorption spectrum with weak LINER-type emission lines or stellar continuum dominated by nebular emission lines of [OII] or [OIII], which are strong compared to any Balmer-line emission. The sample has been cross-correlated with the NVSS 
catalog (Condon et al. 1998). We have converted the flux densities to $5 \mathrm{GHz}$ assuming an $\alpha=0.7$ and corrected for different cosmologies. The resulting RLF is plotted along with the LTS RLF in Fig. 7b.

Because the 2dF/NVSS AGN sample is relatively nearby (median $z=0.2$ ), it allows a direct comparison with the LTS source RLF. We caution, however, that because this sample has been cross-correlated with NVSS data, the radio flux densities may be slightly over-estimated. Figure $7 \mathrm{~b}$ shows that there is an overlap in radio luminosities for the LTS and 2dF/NVSS AGN RLF between the regime $\log P_{5 \mathrm{GHz}} \approx 21$ and $23 \mathrm{~W} \mathrm{~Hz}^{-1}$. In this region of overlap both the normalization and slope of the two RLFs are roughly similar, within the errors. The LTS sources naturally extend the $2 \mathrm{dF} / \mathrm{NVSS}$ AGN RLF to lower luminosities. To emphasize the extreme low powers sampled by our RLF, we note that the lowest power LTS sources are only $\sim 10$ times more powerful than Sgr A* (Melia \& Falcke 2001; see also Falcke et al. 2004). The overall shape and the smooth transition from the 2dF/NVSS AGN RLF to the LTS RLF, suggest a luminosity continuation between these two source populations. It is natural to view the LTS sources as the low-redshift, low-luminosity counterparts of the AGN as sampled by the 2dF/NVSS survey.

\section{Conclusions}

We have undertaken a MERLIN survey of nearby galaxies that did not have available 2 .'5 resolution or better radio observations. Results reveal a $21 \%$ radio-detection rate among the LINER sources, with fifteen radio detections, three of which are new AGN candidates. A compilation of radio observations of all low-luminosity Seyferts, LINERs and composite LINER/HII galaxies in the magnitude-limited Palomar survey reveal a radio-detection rate of $54 \%$ (or $22 \%$ of all bright nearby galaxies), with a more than $50 \%$ detection rate (or $20 \%$ for all bright nearby galaxies) of low-luminosity active nuclei. The radio detection of the Seyferts, LINERs and composite LINER/HII sources in the Palomar survey allow the construction of a local radio luminosity function. Our results show that the Seyferts, LINERs and composite LINER/HII sources form a smooth luminosity transition from higher redshift, more luminous AGN as sampled by the $2 \mathrm{dF} / \mathrm{NVSS}$ survey.

Acknowledgements. M.E.F. acknowledges support from the Fundação para a Ciência e Tecnologia, Ministério da Ciência e Ensino Superior, Portugal through the grant PRAXIS XXI/BD/15830/98 and SFRH/BPD/11627/2002. We are grateful to Jim Ulvestad, Mike Garrett, Simon Garrington, Jim Condon, Naveen Reddy, Marco Spaans and Filippo Fraternali for useful suggestions. Thanks also to Simon Garrington, Anita Richards and Peter Thomasson for valuable help with the data reduction. The authors also wish to acknowledge the anonymous referee for his insightful suggestions.
We have made extensive use of the FIRST and NVSS online database, the NASA/IPAC Extragalactic Database (NED), which is operated by the Jet Propulsion Laboratory, California Institute of Technology, under contract with the National Aeronautics and Space Administration (NASA) and the Automatic Plate Measuring (APM) Facility, run by the Institute of Astronomy in Cambridge. MERLIN is a national facility operated by the University of Manchester on behalf of PPARC.

\section{References}

Anderson, J. M., Ulvestad, J. S., \& Ho, L. C. 2004, ApJ, 603, 42 Antonucci, R. 1993, ARA\&A, 31, 473

Becker, R. H., White, R. L., \& Helfand, D. J. 1995, ApJ, 450, 559

Condon, J. J. 1992, ARA\&A, 30, 575

Condon, J. J., Cotton, W. D., Greisen, E. W., et al. 1998, AJ, 115, 1693

Cowan, J. J., Romanishin, W., \& Branch, D. 1994, ApJ, 436, L139

Falcke, H., Nagar, N. M., Wilson, A. S., \& Ulvestad, J. 2000, ApJ, 542, 197

Falcke, H., Körding, E., Markoff, S. 2004, A\&A, 414, 895

Feretti, L., Giovannini, G., Hummel, E., Kotanyi, G. 1984, A\&A, 137, 362

Filho, M. E., Barthel, P. D., \& Ho, L. C. 2000, ApJS, 129, 93

Filho, M. E., Barthel, P. D., \& Ho, L. C. 2002a, ApJS, 142, 223

Filho, M. E., Barthel, P. D., \& Ho, L. C. 2002b, A\&A, 385, 425

Filho, M. E., Fraternali, F., Markoff, S., et al. 2004, A\&A, 418, 429

Ho, L. C., \& Ulvestad, J. S. 2001, ApJS, 133, 77

Ho, L. C., Filippenko, A. V., \& Sargent, W. L. W. 1995, ApJS, 98, 477

Ho, L. C., Filippenko, A. V., \& Sargent, W. L. W. 1997a, ApJS, 112, 315

Ho, L. C., Filippenko, A. V., \& Sargent, W. L. W. 1997b, ApJ, 487, 568

Ho, L. C., Feigelson, E. D., Townsley, L. K. et al. 2001, ApJ, 549, L51

Hodge, P. W., \& Kennicutt, R. C. 1983, AJ, 88, 296

Huchra, J., \& Burg, R. 1992, ApJ, 393, 90

Kukula, M. J., Pedlar, A., Baum, S. A., \& O’Dea, C. P. 1995, MNRAS, 276, 1262

Melia, F., \& Falcke, H. 2001, ARA\&A, 39, 309

Meurs, E. J. A., \& Wilson, A. S. 1984, A\&A, 136, 206

Nagar, N. M., Falcke, H., Wilson, A. S., \& Ho, L. C. 2000, ApJ, 542, 186

Nagar, N. M., Falcke, H., Wilson, A. S., \& Ulvestad, J. S. 2002, A\&A, 392, 53

Nagar, N. M., Falcke, H., \& Wilson, A. S. 2005, A\&A, 435, 521

Sadler, E., Jackson, C. A., Cannon, R. D., et al. 2002, MNRAS, 329, 227

Schmidt, M. 1968, ApJ, 151, 393

Terashima, Y., \& Wilson, A. S. 2003, ApJ, 583, 145

Terashima, Y., Ho, L. C., \& Ptak, A. F. 2000a, ApJ, 539, 161

Terashima, Y., Ho, L. C., Ptak, A. F., et al. 2000b, ApJ, 533, 729

Turner, J. L., \& Ho, P. T. P. 1994, ApJ, 421, 122

Ulvestad, J. S., \& Ho, L. C. 2001a, ApJ, 558, 561

Ulvestad, J. S., \& Ho, L. C. 2001b, ApJ, 562, L133

Weedman, D. W. 1986, Quasar Astronomy (New York, USA: Cambridge University Press) 